\title{
Parameters of heart rate variability in the critically ill subjects with different disease conditions
}

\author{
M Omerbegovic \\ From ESICM LIVES 2015 \\ Berlin, Germany. 3-7 October 2015
}

\section{Intr}

Heart rate variability has been recognized as a parameter that partly describes autonomic nervous system modulation of cardiovascular system. Analysis of heart rate variability has been proposed as clinically important as predictive and monitoring factor in subjects with different cardiac disease conditions and in subjects who suffer from diabetes mellitus.

Despite numerous clinical and experimental trials on the topic of heart rate variability in the setting of intensive care medicine there is a lack of appropriate technological facilites for routine monitoring and analysis of this phenomenon in everyday clinical practice.

\section{Objectives}

Assessment of the parameters of heart rate variability in critically ill patients with different disease condition.

\section{Methods}

Recordings of forty-two consecutive patients with different disease conditions admitted to intensive care were included in the observational trial. Electrocardiogram was recorded in the periods after admission and primary stabilization of the general condition, and afterwards during the period of stay in intensive care every day (first week of stay). After recording, analysis of short-term segments of electrocardiograms was performed by means of software packages for heart rate vaiability analysis (HrvFreq version 4.0, $\odot 2006$ and Kubios HRV version 2.1,2012). Linear parameters in time domain (mean RR, mean $H R, S D N N$ ) and parameters in frequency domain (TP, LF,HF,LF/HF) and parameters of Poincaré plot (SD1,SD2) were analysed.

\section{Results}

Statistical analysis of the data obtained was performed by software package IBM SPSS version 20. Diversity of the clinical conditions of the patients and different medications that were used on regular bases limited statistical analysis to only descriptive statistics. Results of assessment of different parameters of heart rate variability in the setting of ICU, in small group of subjects with different comorbid states are presented in this paper.

\section{Conclusions}

Subjects in critical state for different disease conditions or after trauma had different patterns of alterations of heart rate variability. In this small group of patients, despite considerable variations in reduction of different parameters of heart rate variability, alterations of heart rate variability were the most pronounced in subjects who had also had developed acute coronary syndrome before admission to the hospital.

\section{Published: 1 October 2015}

\section{References}

1. Schmidt HB, Werdan K, Muller-Werdan U: Autonomic dysfunction in the ICU patient. Curr Opin Crit Care 2001, 7:314-322.

2. Chen WL, Kuo CD: Characteristics of heart rate variability can predict impending septic shock in emergency department patients with sepsis. Acad Emerg Med 2007, 14:392-397.

3. Task Force of the European Society of Cardiology and the North American Society of Pacing and Electrophysiology: Standards of measurement, physiological interpretation and clinical use. Circulation 1996, 93:1043-65.

doi:10.1186/2197-425X-3-S1-A214

Cite this article as: Omerbegovic: Parameters of heart rate variability in the critically ill subjects with different disease conditions. Intensive Care Medicine Experimental 2015 3(Suppl 1):A214. 